\title{
Temperature dependence of enhanced optical absorption and Raman spectroscopy from metallic nanoparticles
}

\author{
C.W. Chen ${ }^{\text {a }}$, H.-P. Chiang ${ }^{\mathrm{a}, \mathrm{b}, *}$, P.T. Leung ${ }^{\mathrm{a}, \mathrm{c}, \mathrm{d}}$, D.P. Tsai ${ }^{\mathrm{d}}$ \\ a Institute of Optoelectronic Sciences, National Taiwan Ocean University, Keelung, Taiwan, ROC \\ ${ }^{\mathrm{b}}$ Institute of Physics, Academia Sinica, Taipei, Taiwan, ROC

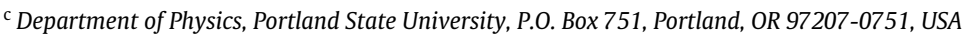 \\ d Department of Physics, National Taiwan University, Taipei 10617, Taiwan, ROC
}

\section{A R T I C L E I N F O}

\section{Article history:}

Received 26 May 2008

Received in revised form

7 August 2008

Accepted 12 September 2008 by R. Merlin

Available online 23 September 2008

\section{PACS:}

78.20.Ci

78.20.Nv

78.30.-j

Keywords:

A. Metallic nanoshells

D. Thermal-optic properties

\begin{abstract}
A B S T R A C T
The temperature dependence of the optical/spectroscopic properties of metallic nanoparticles (in particular nanoshells) including absorption, scattering, and enhanced-Raman scattering for admolecules is studied via theoretical modeling. For core-shell particles, we focus on both the high frequency (symmetric) and low frequency (antisymmetric) plasmon resonance modes of the nanoshells. It is found, that while these properties in general have optimal values at a certain temperature specific to the shell configuration except for relatively thin shells, the SERS enhancement generally has more appreciable variations and decreases when the nanoshells get heated up from room temperature. Implications for various applications with these nanoparticles are discussed.
\end{abstract}

(C) 2008 Elsevier Ltd. All rights reserved.

\section{Introduction}

Among the many plasmonic systems recently developed, metallic nanoparticles - in particular metallic nanoshells - have been recognized as one of the most versatile systems due to its high tunability in optical resonance and its capability of creating strongly-enhanced local electromagnetic fields. Since its first fabrication in 1998 [1], the nanoshell system has been studied intensively for the past decade, with focus both on its fundamental optical properties and in its large variety of applications: from enhanced spectroscopy to photothermal cancer therapy [2]. Experimentally, both dielectric [3,4] and hollow [5, 6 ] cores can be fabricated using methods of colloidal chemistry. Furthermore, by varying the metal shell thickness towards the thin shell limit (in the order of a few $\mathrm{nm}$ ), the plasmon frequency can be adjusted across the whole visible spectrum - from near UV to near IR [3].

Over the years, contributions from both theoretical and experimental studies have revealed many aspects of these shells

\footnotetext{
* Corresponding author at: Institute of Optoelectronic Sciences, National Taiwan Ocean University, Keelung, Taiwan, ROC.

E-mail addresses: hpchiang@mail.ntou.edu.tw (H.-P. Chiang), hopl@pdx.edu (P.T. Leung).
}

such as their optical absorption and extinction properties [7]; their role in surface enhanced Raman scattering (SERS) [8$10]$; etc. However, these studies have been mostly carried out at room temperatures; and a systematic investigation on how these nanoshell properties vary as a function of environmental temperature seems to have been lacking thus far. It is expected that these temperature effects could be significant just as in the case with ordinary metallic nanoparticles, in which the SERS effect is largely depreciated at elevated temperatures [11]. One possible significance of this temperature effect with the metallic nanoshell can take place in its application in cancer therapy employing photothermal effects, since in this process the heat generated (which is used to destroy cancer cells) will in turn affect possibly the absorption peak of these shells to make it go off-resonance and thus affecting the therapeutic efficiency [2].

It is our purpose in the present study to present a theoretical model which simulates these temperature effects to reveal how the optical absorption and enhanced SERS by these nanoshells will be affected, and we shall focus on each of the two (symmetric and antisymmetric) plasmon resonance modes of the coreshell particle. A recent work has also carried out a modeling study on the thermalplasmonic effects for a spheroidal coreshell particle [12]. However, this work was limited only to the calculation of extinction for the "low frequency mode" since the 
splitting into symmetric and antisymmetric modes for the prolate spheroidal particle studied was not obvious with the dimensions studied in Ref. [12]. Furthermore, the temperature effects on SERS from these nanoshells were not studied previously and also the semiconducting core used in [12] can have complicated temperature effects not accounted for in the previous modeling. Here we shall devote our study to spherical nanoshells with an insulating core so that the temperature effects will be mainly due to the metal shells and the splitting into the high and low plasmonic resonance modes is distinctly obvious. We shall limit ourselves to a classical phenomenological optothermal model and a modest temperature range from $100 \mathrm{~K}$ to $400 \mathrm{~K}$, over which the nanoshell structure will likely remain stable.

\section{Theoretical model}

To construct a model which will describe the temperature dependence of the optical absorption, scattering, and SERS effect from a metallic nanoshell, we shall employ several of our previously-published works for the present simulation. Since the nanoshell sizes we consider will be much smaller than the optical wavelengths, we shall adopt a long-wavelength approximation within which the optical response of the nanoshell can be completely accounted for using the following multipole polarizability of the shell [9]:

$$
\begin{aligned}
& \alpha_{\ell}^{S} \\
& =\frac{\left(\varepsilon-\varepsilon^{\prime}\right) \ell[\ell+\varepsilon(\ell+1)] a^{2 \ell+1}+\ell(1-\varepsilon)\left[\varepsilon^{\prime} \ell+\varepsilon(\ell+1)\right] b^{2 \ell+1}}{\left(\varepsilon-\varepsilon^{\prime}\right)(\varepsilon-1) \ell(\ell+1) a^{2 \ell+1}-(\varepsilon \ell+\ell+1)\left[\varepsilon^{\prime} \ell+\varepsilon(\ell+1)\right] b^{2 \ell+1}} b^{2 \ell+1},
\end{aligned}
$$

where we have assumed a nanoshell of dimension $a$ (inner radius) and $b$ (outer radius), and the shell is made of a metal of dielectric function $\varepsilon$ coated on an insulator core of dielectric constant $\varepsilon^{\prime}$. To study how light (of wavelength $\lambda$ ) interacts with these nanoshells, we shall calculate the absorption and scattering efficiencies of these systems which can be expressed in terms of the dipole polarizability $\left(\alpha_{\ell}^{S}=\alpha_{1}^{S}\right)$ of the shells as follows:

$Q_{a b}=\frac{8 \pi}{\lambda b^{2}} \operatorname{Im}\left(\alpha_{1}^{S}\right)$,

$Q_{s c}=\frac{8}{3}\left(\frac{2 \pi}{\lambda}\right)^{4}\left|\frac{\alpha_{1}^{S}}{b}\right|^{2}$,

and the sum of which will give the total extinction coefficient of the nanoshell. To study enhanced molecular spectroscopy induced by these nanoshells, we shall consider the following SERS enhancement ratio $[9,11]$ :

$R=\left|\frac{1}{1-\alpha G_{\perp}}\left(1+\frac{2 \alpha_{1}^{S}}{(b+d)^{3}}\right)\right|^{4}$,

where we have assumed a molecular dipole of polarizability $\alpha$ located along the radial direction and at a distance $d$ from the shell, and the "image factor" in (4) is given by:

$G_{\perp}=\sum_{\ell} \alpha_{\ell}^{S} \frac{(\ell+1)^{2}}{(b+d)^{2 \ell+4}}$.

To study the effects of temperature on the quantities in Eqs. (2)(4), we have to account for (a) the temperature dependence of the metal dielectric function $\varepsilon$, and (b) the expansion/contraction of the shell dimensions.

To account for the effect in (a), we briefly summarize a previous model we established to describe these effects [13]. To begin, we describe the dielectric response of metal using the Drude model:

$\varepsilon=1-\frac{\omega_{P}^{2}}{\omega\left(\omega+\mathrm{i} \omega_{c}\right)}$, where $\omega_{c}$ is the collision frequency and $\omega_{p}$ the plasma frequency given by:

$\omega_{p}=\sqrt{\frac{4 \pi N e^{2}}{m^{*}}}$,

with $N$ and $m^{*}$ the density and effective mass of the electrons, respectively. Hence, assuming the variation of $m^{*}$ with $T$ can be ignored [13], $\omega_{p}$ depends on $T$ via volumetric effects as follows [14]:

$\omega_{p}=\omega_{p 0}\left[1+\gamma\left(T-T_{0}\right)\right]^{-1 / 2}$,

where $\gamma$ is the expansion coefficient of the metal, and $T_{0}$ is a reference temperature taken to be the room temperature. The collision frequency will have contributions from both phonon-electron and electron-electron scattering, as well as a surface scattering term from the two shell boundaries:

$\omega_{c}=\omega_{c p}+\omega_{c e}+\omega_{s}$,

and can be modeled using the various scattering models in the literature. We thus obtain [13]:

$\omega_{c p}(T)=\omega_{0}\left[\frac{2}{5}+4\left(\frac{T}{\theta}\right)^{5} \int_{\theta}^{\theta / T} \frac{z^{4} \mathrm{~d} z}{\mathrm{e}^{z}-1}\right]$,

where $\theta$ is the Debye temperature and $\omega_{0}$ is a constant to be determined from the static limit of the above expression together with the knowledge of the d.c. conductivity. In addition, we have:

$\omega_{c e}(T)=\frac{1}{12} \pi^{3} \frac{\Gamma \Delta}{\hbar E_{F}}\left[\left(k_{B} T\right)^{2}+(\hbar \omega / 2 \pi)^{2}\right]$,

where $\Gamma$ and $\Delta$ are constants as described in [13]; and

$\omega_{s}=\frac{\mathrm{Av}_{F}}{[b(T)-a(T)]}$,

with $v_{F}$ being the Fermi velocity of the metal, a geometrical factor $A$ of unity order of magnitude, and the shell radii as functions of temperature given by $b(T)=b_{0}\left[1+\frac{\gamma}{3}\left(T-T_{0}\right)\right]$, etc., in terms of the volume expansivity of the metal. Thus Eqs. (6)-(12) provide a model for the temperature dependence of $\varepsilon$, which when used in (1) will describe the temperature variation of the multipole polarizability of the nanoshell. This then will predict how the various quantities in (2)-(4) vary with temperature.

\section{Numerical results and discussion}

We have performed some numerical studies of the temperature effects on the quantities in Eqs. (2)-(4) for a silver nanoshell using the parameters given in Ref. [13].

Fig. 1 shows frequency spectra of the quantities in Eqs. (2)(4) at room temperature $(T=300 \mathrm{~K})$ for a silver shell of aspect ratio $(b / a)=50 \mathrm{~nm} / 40 \mathrm{~nm}$. The two resonances (i.e. symmetric and antisymmetric modes) are clearly seen from all the spectra. This arises from the greater restoring force (provided by the positive background in the jellium model) present in the antibonding (symmetric) modes which in turn leads to a higher resonance frequency compared to that for the bonding (antisymmetric) modes [15]. This is in complete analogy to the so-called long- and short-range plasmons first discovered in 1981 from the cross-coupling of the two interfacial plasmons across a planar thin metal film [16]. We also see that scattering generally dominates over absorption in the extinction process for incident light onto this relatively large nanoshells. Moreover, appreciable SERS enhancement $\left(\sim 10^{6}\right)$ can be obtained at frequency close to that for the bonding mode. Next, we study the temperature effects on these quantities. Figs. 2 and 3 show that while all 


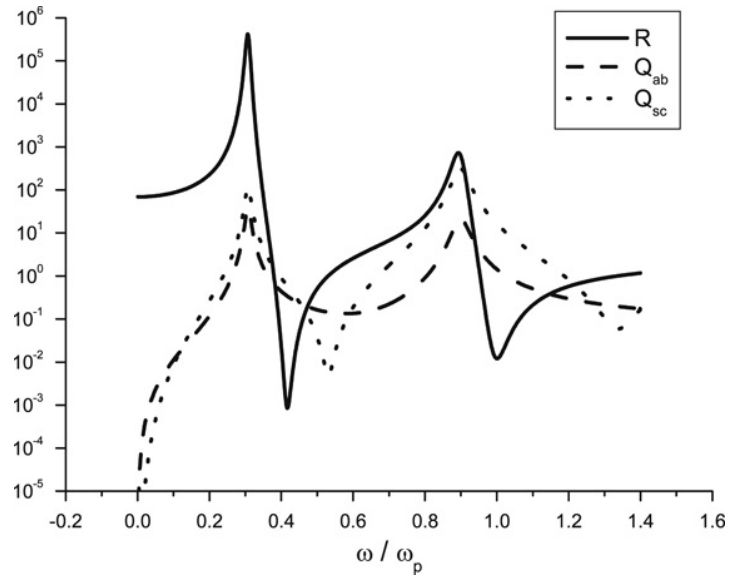

Fig. 1. SERS enhancement ratio $(R)$, absorption efficiencies $\left(Q_{a b}\right)$ and scattering efficiencies $\left(Q_{s c}\right)$ of a silver shell with aspect ratio $(b / a)=50 \mathrm{~nm} / 40 \mathrm{~nm}$ at room temperature $(T=300 \mathrm{~K})$.

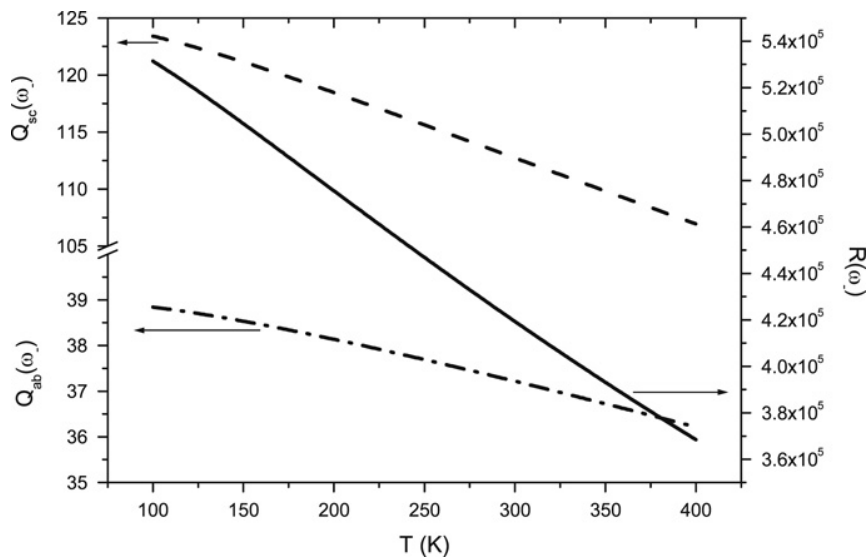

Fig. 2. Temperature dependence of the peak values for the SERS enhancement ratio, absorption efficiencies, and scattering efficiencies of a silver shell with aspect ratio $(b / a)=50 \mathrm{~nm} / 40 \mathrm{~nm}$ at the low resonance frequency $\left(\omega_{-}=0.308 \omega_{\mathrm{P}}\right)$.

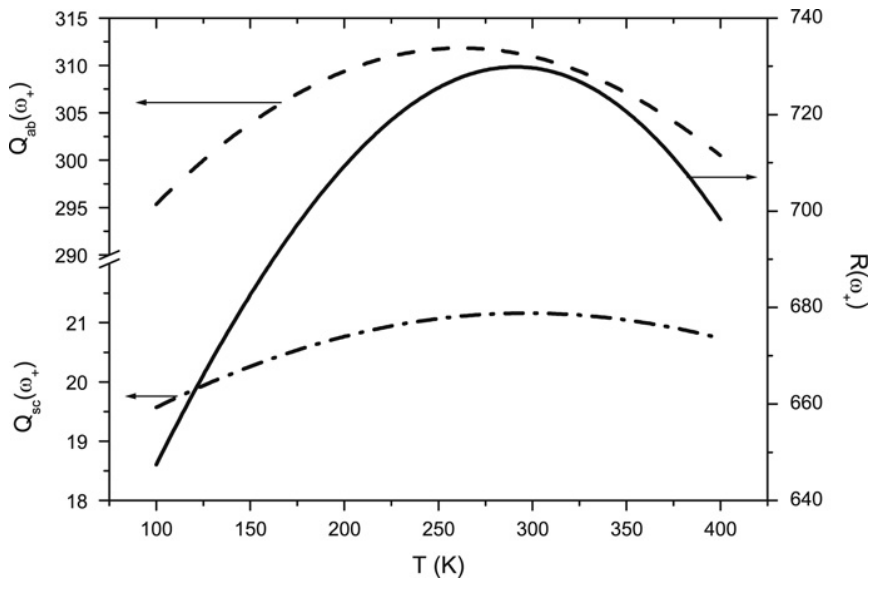

Fig. 3. Same as in Fig. 2, but for values at the high resonance frequency $\left(\omega_{+}=\right.$ $\left.0.899 \omega_{P}\right)$.

the quantities decrease more or less monotonically with the increase of temperature at the low resonance frequency case, there exists an "optimal temperature" ( $\sim 250-300 \mathrm{~K})$ for them to acquire maximum values in the high resonance frequency case. The interesting implication is that in either case, all of them will become less efficient if the nanoshell temperature starts to increase from room temperature. This finding may have relevance

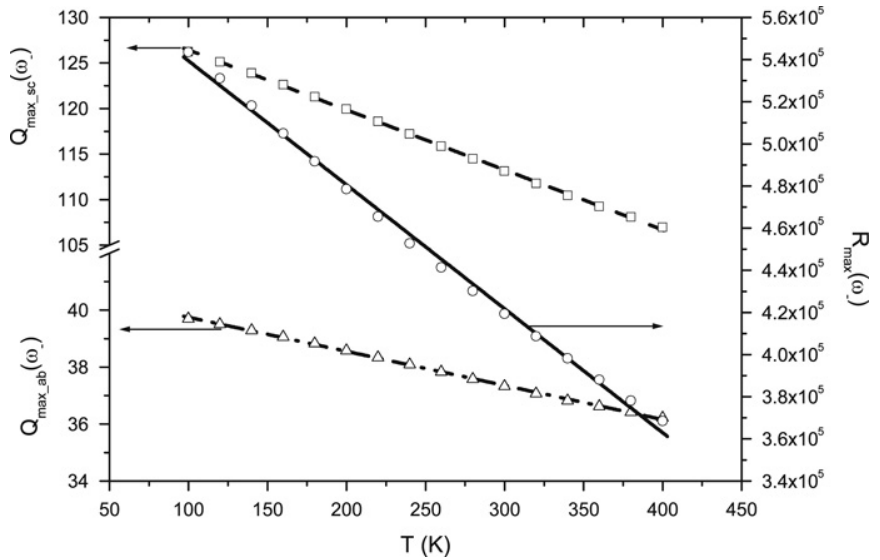

Fig. 4. Temperature variation of the maximum possible peak values of the SERS enhancement ratio, absorption efficiencies and scattering efficiencies for the silver shell in Fig. 2 at the low resonance frequency.

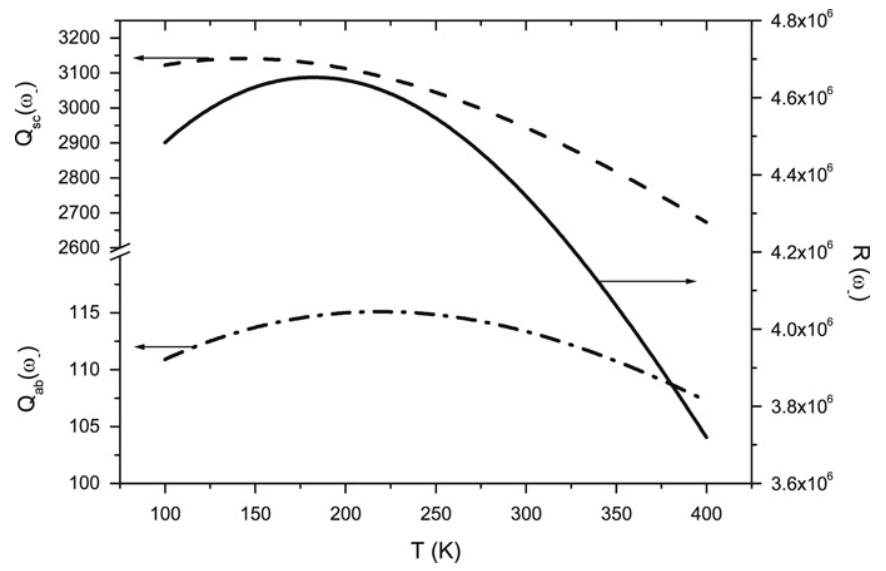

Fig. 5. Same as in Fig. 2, but for a silver shell with aspect ratio $(b / a)=$ $50 \mathrm{~nm} / 20 \mathrm{~nm}$ at the low resonance frequency $\left(\omega_{-}=0.517 \omega_{P}\right)$.

for the application of these nanoshells in cancer therapy utilizing the heat generated via resonant absorption of near IR radiation [2]. However, since the resonance frequencies are actually changing with temperatures, it would be more interesting to study the maximum possible values of these quantities at one temperature. Fig. 4 plots how these peak values at the low resonance frequency vary with temperature, and it is seen that an almost monotonic decrease of these quantities takes place as temperatures increases. A similar phenomenon is observed (not shown) for these peak values at the high frequency resonance, except that the SERS ratio is almost 3 orders of magnitude smaller at the symmetric modes for this nanoshell. Next we study the same effects for a thicker nanoshell with aspect ratio $50 \mathrm{~nm} / 20 \mathrm{~nm}$. The results shown in Figs. 5-7 behave quite similar to those in Figs. 2-4 for the thinner nanoshell, except that all the three quantities at frequency close to the low resonance frequency no more decrease monotonically with the rise of temperature. Instead, there exist optimum temperatures for these quantities at frequencies closed to both the high and low resonance frequency. Moreover, the values of all the three quantities are in general higher than those for the thinner shell. One interesting implication from these results is that if one intends to increase these quantities by lowering the nanoshell temperature, one has to be aware of the existence of a certain "critical temperature" below which these quantities will start to decrease with the lowering of temperatures - except for thin shells at frequencies close to that of the antisymmetric modes. We have further study the same phenomena with a metallic nano particle of the same material for various dimensions, and have 


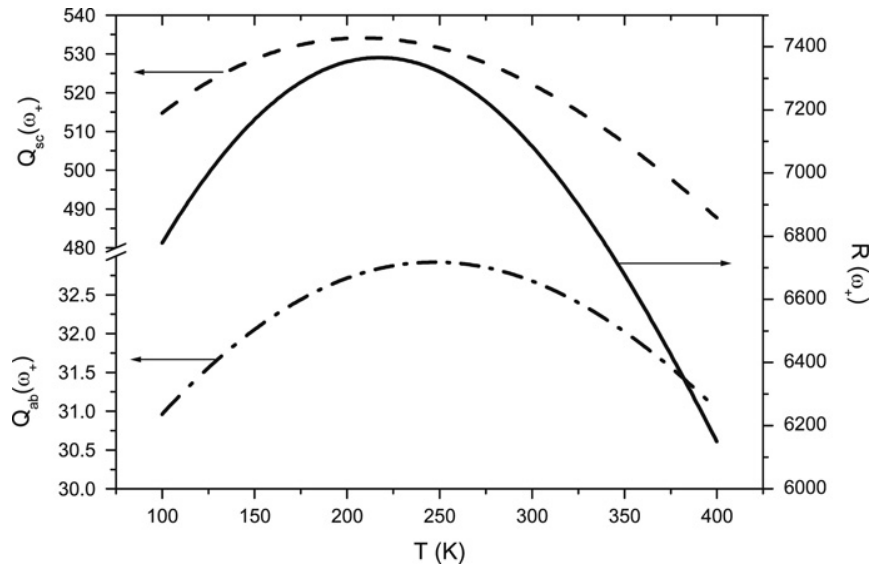

Fig. 6. Same as in Fig. 5, but for values at the high resonance frequency $\left(\omega_{+}=\right.$ $\left.0.741 \omega_{P}\right)$.

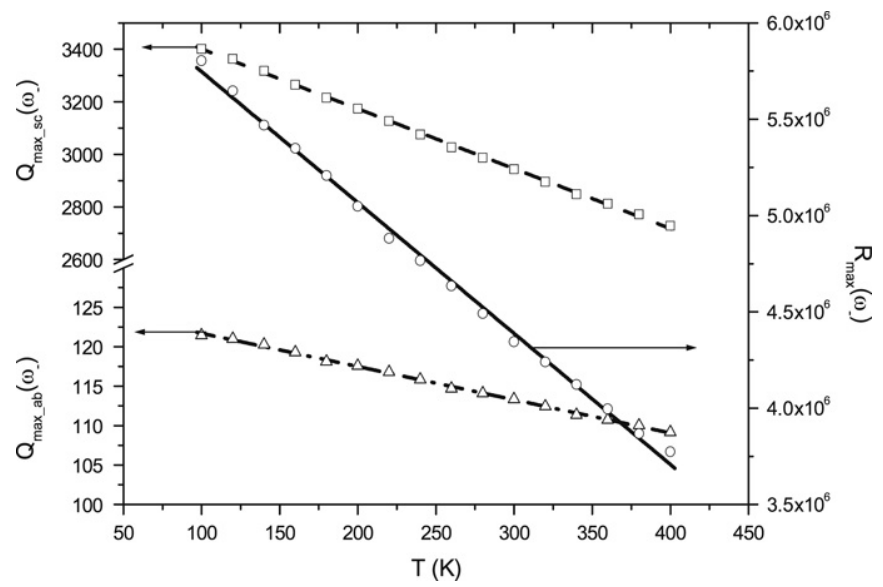

Fig. 7. Temperature variation of the maximum possible peak values of the SERS enhancement ratio, absorption efficiencies and scattering efficiencies for the silver shell in Fig. 5 at the low resonance frequency.

found results very similar to those for the thick shell (i.e. the one with $a / b=50 \mathrm{~nm} / 20 \mathrm{~nm}$ ), as shown in Fig. 8 . These results are obtained by setting an exceedingly small value $(\sim 0.5 \mathrm{~nm})$ for the inner radius. While experimental testing of these results may be relatively easier, it seems to imply that there may exist a critical value for the inner shell radius, above which all quantities will optimize their values at a certain temperature. This information will be useful for one to try to optimize these quantities in various applications via the control of the nanoshell temperature.

\section{Conclusion}

In this work, we have studied the temperature dependence of various enhanced optical processes using metallic nanoparticles and shells. These include optical absorption, scattering, and SERS from shells of two different thicknesses as well as for a solid particle. It was found that in general all these optical/spectroscopic efficiencies decrease as temperature rises from that at room temperature. However, such efficiencies will increase at lower temperatures - reaching an optimum value except for the thin shells in which case the increase could be monotonic at optical frequencies close to the symmetric SP mode of the nanoshell. The

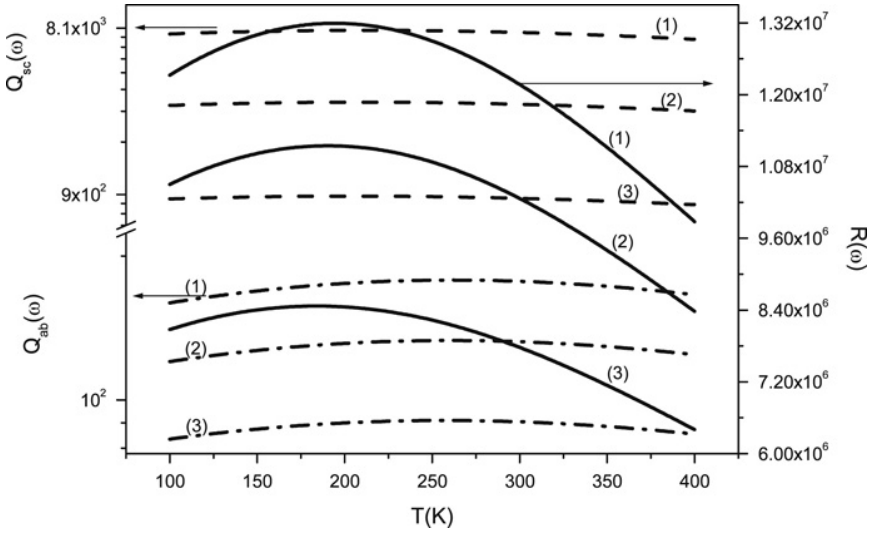

Fig. 8. Temperature dependence of the peak values for the SERS enhancement ratio, absorption efficiencies, and scattering efficiencies of a silver shell with aspect ratio $(b / a)=$ (1) $50 \mathrm{~nm} / 0.5 \mathrm{~nm}$, (2) $40 \mathrm{~nm} / 0.5 \mathrm{~nm}$, (3) $30 \mathrm{~nm} / 0.5 \mathrm{~nm}$ at the resonance frequency $\left(\omega=0.577 \omega_{P}\right)$, respectively.

physical explanation for such temperature-dependence to arise is complicated, and depends on the coupling efficiency between the "surface mode" and the "cavity mode" across the metal shell as temperature varies. While the effects on absorption may be significant for applications such as in the near IR thermal therapy using these nanoshells; those on SERS may have useful implications to a broader class of possible catalytic photochemical processes employing the SP enhancements from these nanoshells. Future experiments conducted to investigate these temperature effects will be of interest.

\section{Acknowledgments}

H.-P. Chiang acknowledges financial support from Center for Marine Bioscience and Biotechnology, National Taiwan Ocean University and the National Science Council of ROC under grant numbers NSC 97-2112-M-019-001-MY3 and NSC 96-2622-M-019 $-001-C C 3$. P. T. Leung acknowledges supports from the National Science Council of ROC under grant number NSC-97-2811-M002-018, the Fulbright Foundation, as well as the PSU Faculty Enhancement Grant. D. P. Tsai acknowledges financial support from National Science Council of ROC under grant number NSC 972120-M-002-013 and NSC 96-2923-M-002-002-MY3.

\section{References}

[1] S.J. Oldenburg, R.D. Averitt, S.L. Westcott, N.J. Halas, Chem. Phys. Lett. 288 (1998) 243.

[2] For a recent review, especially on biomedical applications of nanoshells, see L.R. Hirsch, et al., Ann. Biomed. Eng. 34 (2006) 15.

[3] S.J. Oldenburg, S.L. Westcott, R.D. Averitt, N.J. Halas, J. Chem. Phys. 111 (1999) 4729.

[4] S.L. Westcott, J.B. Jackson, C. Radloff, N.J. Halas, Phys. Rev. B 66 (2002) 155431.

[5] C. Graf, A.v. Blaaderen, Langmuir 18 (2002) 524.

[6] Y. Sun, Y. Xia, Anal. Chem. 74 (2002) 5297.

[7] K.L. Kelly, E. Coronado, L.L. Zhao, G.C. Schatz, J. Phys. Chem. B 107 (2003) 668

[8] J.B. Jackson, S.L. Westcott, L.R. Hirsch, J.L. West, N.J. Halas, Appl. Phys. Lett. 82 (2003) 257.

[9] Z.E. Goude, P.T. Leung, Solid State Commun. 143 (2007) 416

[10] F. Le, et al., ACS Nano 2 (2008) 707.

[11] P.T. Leung, M.H. Hider, E.J. Sanchez, Phys. Rev. B 53 (1996) 12659.

[12] N.J. Florous, K. Saitoh, M. Koshiba, IEEE Trans. Nanotechnol. 6 (2007) 549

[13] H.P. Chiang, P.T. Leung, W.S. Tse, Solid State Commun. 101 (1997) 45.

[14] H.P. Chiang, Y.C. Wang, P.T. Leung, W.S. Tse, Opt. Commun. 188 (2001) 283.

[15] R. Chang, P.T. Leung, Phys. Rev. B 73 (2006) 125478; Phys. Rev. B 75 (2007) 079901(E).

[16] D. Sarid, Phys. Rev. Lett. 47 (1981) 1927. 